\title{
The structure of field line resonances in a dipole magnetosphere with moving plasma
}

\author{
D. A. Kozlov and A. S. Leonovich \\ Institute of Solar-Terrestrial Physics (ISTP), Russian Academy of Sciences, Siberian Branch, Irkutsk, Russia \\ Received: 16 July 2007 - Revised: 9 January 2008 - Accepted: 19 February 2008 - Published: 26 March 2008
}

\begin{abstract}
A theoretical model of field line resonance in the magnetosphere with a dipole magnetic field and moving plasma was constructed. Motion of medium was simulated by its azimuthal rotation. Analytical and numerical solutions were found for MHD equations describing the structure of field line resonances. It was shown that a monochromatic, fast magnetosonic wave can excite several harmonics of standing Alfvén waves on different resonant surfaces at once. The resonant surface density is maximum in the transition layer at the magnetopause. The resonant oscillation amplitude profile becomes asymmetric in our model in regions with a maximum gradient of the background plasma velocity (plasmapause and magnetopause), in contrast to models with immobile plasma. In the same regions, the phase of resonant oscillations varies non-monotonically across the resonant surface which may serve as a detector of such regions in geomagnetic pulsation observations.
\end{abstract}

Keywords. Magnetospheric physics (MHD waves and instabilities; Planetary magnetospheres) - Radio science (Magnetospheric physics)

\section{Introduction}

Resonant Alfvén waves are a successful concept for investigating magnetospheric MHD oscillations (Dungey, 1954), known as field line resonance (FLR). Tamao (1965) pointed out the possibility of resonant excitation of Alfvén oscillations by a monochromatic fast magnetosonic (FMS) wave. This idea was further developed by Radoski (1974); Chen and Hasegawa (1974); Southwood (1974) and others.

There is a close connection between resonant Alfvén oscillations and different types of aurora (Samson et al., 1996;

Correspondence to: A. S. Leonovich

(leon@iszf.irk.ru)
Lysak and Song, 2003). Kinetic Alfvén waves possess a longitudinal (about the magnetic field) component of the electric field capable of accelerating charged magnetospheric particles precipitating in the ionosphere and induce optical aurorae (Hasegawa, 1976; Goertz, 1984).

A theory of magnetospheric MHD oscillations was first developed for simple models of the magnetosphere. Kivelson and Southwood (1986); Southwood and Kivelson (1986) employed a box model with straight magnetic field lines that were bounded by two opposite planes which simulated the high-conductivity ionosphere. This model enables us to explore the FLR phenomenon, taking into account plasma inhomogeneity, both along and across the magnetic field. Allan et al. $(1986,1987)$ used a hemicylindrical magnetospheric model in which magnetic field lines were semicircles. This model provided an opportunity for investigating the curvature effect of magnetic field lines in FLR.

The next step was to use a dipole magnetic field (Lifshitz and Fedorov, 1986; Leonovich and Mazur, 1989; Chen and Cowley, 1989; Lee and Lysak, 1989). These studies explored the curvature effects of magnetic field lines and plasma inhomogeneities along magnetic field lines and across magnetic shells. They were generalized to the case of threedimensional plasma in which the plasma inhomogeneity in the azimuthal direction is also taken into account (Lee et al., 1989). One of the distinctive features in dipole-like magnetospheric models is the multiplicity of resonances that can be excited by the monochromatic magnetosonic wave within the magnetosphere (Leonovich, 2001). In this case, the highest concentration of resonant shells is in the layer adjoining the magnetopause.

All the studies mentioned above concerned magnetospheric models with immobile plasma. It is well known that regions of high gradients of the plasma velocity, which can be a source of resonant Alfvén waves, create conditions where the Kelvin-Helmholtz instability may develop (McKenzie, 1970; Leonovich and Mishin, 2005). In the

Published by Copernicus Publications on behalf of the European Geosciences Union. 


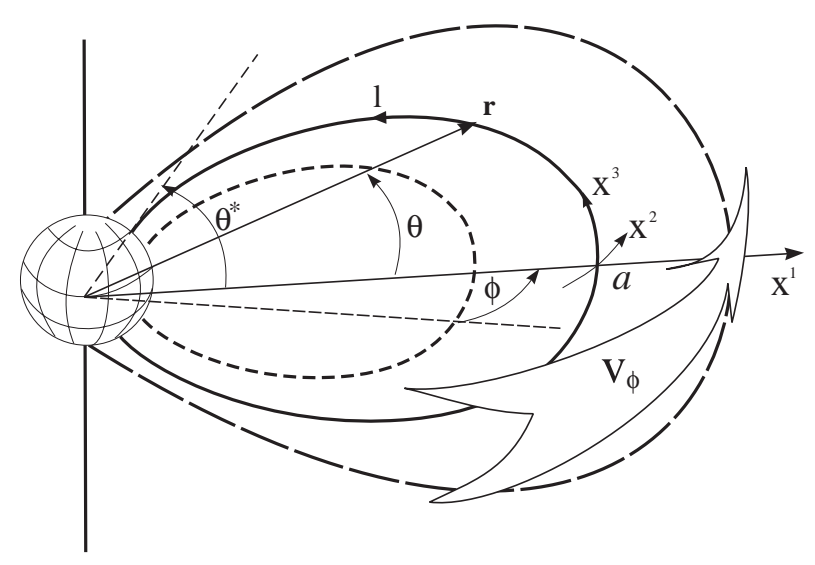

Fig. 1. A magnetospheric model with dipole magnetic field and azimuthally rotating plasma $\left(\boldsymbol{v}=\left(0, v_{\phi}, 0\right)\right)$. Coordinate systems tied to magnetic field lines are shown: a curvilinear orthogonal system $\left(x^{1}, x^{2}, x^{3}\right)$ and a non-orthogonal system of coordinates $(a, \phi, \theta)$, used in numerical calculations.

Earth's magnetosphere, such conditions can be realized on the magnetopause and plasmapause. Hence the plasma motion may be an important factor influencing FLR. A recent study by Walker (2005) considers the possibility of exciting FLR by a magnetosonic wave that passes from the solar wind to the magnetosphere through a magnetopause with shear flow. In this study an elementary, inhomogeneous, one-dimensional slab model of the magnetosphere at rest in the homogenous moving plasma of the solar wind was employed.

In this paper, our concern is with the effect of plasma motion on the FLR structure in the dipole magnetosphere. We used a model in which plasma motion is simulated by its azimuthal rotation. In this model, the plasma is assumed to be moving both within (at convective velocity) and outside the magnetosphere (at solar wind velocity). Of particular importance is the transition zone between the magnetosphere and the solar wind near the plasmapause, where the plasma motion gradient, and hence its effect on the FLR, are maximum. Here we take no notice of magnetosonic oscillations which are the source of resonant Alfvén waves. We simulated their behaviour by a specially selected driver in the equation for Alfvén waves. In the following, we are going to replace this model driver with an equation solution that describes the FMS wave structure obtained in this study.

The structure of this paper is as follows. Section 2 describes a model of medium and basic equations derived there, which show FLR in the dipole magnetosphere with rotating plasma. The structure of standing Alfvén waves along geomagnetic field lines is examined in Sect. 3. In Sect. 4, an analytical solution was derived for an equation that describes the structure of resonant Alfvén oscillations across magnetic shells. In Sect. 5, we investigate numerically the field of resonant oscillations excited by a monochromatic source in the magnetosphere. This source simulates an FMS wave that penetrates into the magnetosphere from the magnetopause. The principal results obtained here are listed in the Conclusion.

\section{Model of medium and major equations}

In order to solve the problem posed, let us employ a magnetospheric model with a dipole magnetic field and azimuthally rotating plasma (Fig. 1). A self-consistent analytical model of such a magnetosphere was presented in Leonovich et al. (2004). The rotating plasma simulates both its convective motion inside the magnetosphere and in the solar wind flowing round the magnetosphere. Whereas plasma in the process is affected by centrifugal forces, the equilibrium of the plasma configuration is sustained by a gas-kinetic pressure gradient. This rather simple model allows for a realistic description of the distribution of plasma parameters in the dayside magnetosphere.

To examine Alfvén oscillations in this model, we will use the system of ideal MHD equations:

$$
\begin{aligned}
\rho \frac{d \boldsymbol{v}}{d t} & =-\nabla P+\frac{1}{4 \pi}[\operatorname{curl} \boldsymbol{B} \times \boldsymbol{B}], \\
\frac{\partial \boldsymbol{B}}{\partial t} & =\operatorname{curl}[\boldsymbol{v} \times \boldsymbol{B}], \\
\frac{\partial \rho}{\partial t} & +\left(\nabla_{1}-(\ln \bar{\omega})^{\prime}\right)=0, \\
\frac{d}{d t} \frac{P}{\rho^{\gamma}} & =0
\end{aligned}
$$

where $\boldsymbol{B}$ and $\boldsymbol{v}$ are the magnetic field vectors and plasma motion velocities, $P$ and $\rho$ are the plasma pressure and density, $\gamma$ is the adiabatic index. In Eqs. (1) and (4), $d / d t=\partial / \partial t+(v \nabla)$ represents the Lagrangian derivative in moving plasma. In steady state $(\partial / \partial t=0)$, the set of Eqs. (14) describes the distribution of the plasma equilibrium parameters $\boldsymbol{B}_{0}, \boldsymbol{v}_{0}, P_{0}$ and $\rho_{0}$.

Let us introduce a curvilinear orthogonal coordinate system $\left(x^{1}, x^{2}, x^{3}\right)$ tied to magnetic field lines. The $x^{3}$ coordinate is directed along the field line, $x^{1}$ across magnetic shells, whereas $x^{2}$ is directed azimuthally, resulting in a right-handed coordinate system. In this system of coordinates $\boldsymbol{B}_{0}=\left(0,0, B_{03}\right), \boldsymbol{v}_{0}=\left(0, v_{02}, 0\right)$, while the length element has the form

$d s^{2}=g_{1}\left(d x^{1}\right)^{2}+g_{2}\left(d x^{2}\right)^{2}+g_{3}\left(d x^{3}\right)^{2}$,

where $g_{i}(i=1,2,3)$ are metrical tensor elements. Using the azimuthal angle $\phi$ as the azimuthal coordinate yields $v_{02} \equiv v_{\phi}=\sqrt{g_{2}} \Omega$, where $\Omega$ is the angular velocity of rotating plasma. Plasma rotation on each magnetic shell has a constant angular velocity $\Omega \equiv \Omega\left(x^{1}\right)$. The magnetospheric portion corresponding to the plasmasphere is immobile. In the outer magnetosphere, the velocity of plasma rotation 
reaches $v_{02} \sim 30-50 \mathrm{~km} / \mathrm{s}$; this value is characteristic for convective plasma motion in the outer magnetosphere. Passing through the magnetopause, the plasma velocity increases to $v_{02} \approx 400 \mathrm{~km} / \mathrm{s}$, typical of the solar wind.

Next, we linearise the system of Eqs. (1-4) relative to small disturbances due to the plasma MHD oscillations. Consider the monochromatic oscillations of the form $\exp \left(-i \omega t+i k_{2} x^{2}\right)$, where $\omega$ is the oscillation frequency, $k_{2}$ is the azimuthal wave vector (if $x^{2}=\phi$ is the azimuthal angle, then $k_{2} \equiv m=0, \pm 1, \pm 2, \ldots$ is the azimuthal wave number). The first two Eqs. (1) yield

$$
\begin{gathered}
-\rho_{0}\left(i \bar{\omega} v_{1}+v_{2} \Omega \nabla_{1} \ln g_{2}\right)-\frac{\tilde{\rho} \Omega^{2}}{2} \nabla_{1} g_{2}= \\
-\nabla_{1} \widetilde{P}-\frac{B_{0}}{4 \pi} \frac{1}{\sqrt{g_{3}}}\left(\nabla_{3} B_{1}-\nabla_{1} B_{3}\right), \\
\rho_{0}\left(-i \bar{\omega} v_{2}+v_{1} \frac{\nabla_{1}\left(g_{2} \Omega\right)}{g_{1}}+\frac{v_{3} \Omega}{g_{3}} \nabla_{3} g_{2}\right)= \\
-i k_{2} \widetilde{P}-\frac{B_{0}}{4 \pi} \frac{1}{\sqrt{g_{3}}}\left(i k_{2} B_{3}-\nabla_{3} B_{2}\right),
\end{gathered}
$$

where $\nabla_{i} \equiv \partial / \partial x^{i} \quad(i=1,2,3)$, while $v_{i}$ and $B_{i}$ are the disturbed velocity and disturbed magnetic field vector components, $\widetilde{P}$ and $\widetilde{\rho}$ are the disturbed pressure and density of plasma. The added notation $\bar{\omega}=\omega-m \Omega$ here refers to the Doppler-shifted oscillation frequency in the rotating plasma.

To describe MHD oscillations, it is convenient to switch from the components of the electromagnetic field and disturbed velocity field to potentials. According to the Helmholtz expansion theorem (Korn and Korn, 1968), an arbitrary vector field can be represented as the sum of the vortex-free and solenoidal fields.

Let us represent the disturbed electric field of the oscillations as

$\mathbf{E}=-\nabla \varphi+\operatorname{curl} \Psi$,

where $\varphi$ is the scalar potential, and $\Psi=\left(\psi_{1}, \psi_{2}, \psi_{3}\right)$ is the vector potential. This obviously implies that $\mathbf{E}$ is invariant to an arbitrary constant added to the scalar potential $\varphi \rightarrow \varphi+$ const and to an arbitrary gradient added to the vector potential $\Psi \rightarrow \Psi+\nabla \chi$. Without losing generality one can choose const $=0$, while selecting $\nabla \chi$ such that $\psi_{1}+\nabla_{1} \chi=0$, i.e. $\Psi=(0, \xi, \psi)$, where $\xi=\psi_{2}+\nabla_{2} \chi, \psi=\psi_{3}+\nabla_{3} \chi$.

The electric, $\mathbf{E}$, magnetic, $\boldsymbol{B}$, and velocity, $\boldsymbol{v}$, fields of oscilaltions are discribed by Eq. (2) and

$\mathbf{E}=-\frac{1}{c}\left(\boldsymbol{v} \times \boldsymbol{B}_{0}+\boldsymbol{v}_{0} \times \boldsymbol{B}\right)$,

which allows the components of $\boldsymbol{B}$ and $\boldsymbol{v}$ to be expressed in terms of the potentials $\varphi, \xi$ and $\psi$. Based on Eqs. (3) and (4), one can also express the disturbed density $\widetilde{\rho}$ and pressure $\widetilde{P}$ in terms of the potentials.
Substituting these expressions into Eqs. (5), (6), we deduce the following system of equations:

$$
\begin{aligned}
& \nabla_{1} \widehat{L}_{T}\left(\nabla_{1}-(\ln \bar{\omega})^{\prime}\right) \varphi-k_{2}^{2} \widehat{L}_{P} \varphi=i k_{2} \hat{L}_{F 0} \psi+i \hat{L}_{F 1} \psi, \\
& \frac{S^{2}}{C_{s}^{2}}\left[\frac{g_{3}}{\sqrt{g}} \nabla_{1} \frac{g_{2}}{\sqrt{g}} \nabla_{1} \psi-\frac{k_{2}^{2}}{g_{2}} \psi-\frac{k_{2} \Omega}{\bar{\omega}} \frac{\nabla_{3}^{2} \psi}{g_{3}}\right]+ \\
& \sqrt{g_{3}} \hat{L}_{T}(\bar{\omega}) \frac{g_{1}}{\sqrt{g}} \\
& {\left[\psi+\frac{\Omega g_{2}}{k_{2} \bar{\omega}}\left(\frac{\omega}{\bar{\omega}}(\ln \Omega)^{\prime} \frac{\nabla_{1} \psi}{g_{1}}+\frac{\nabla_{1}^{2} \psi}{g_{1}}+\frac{\nabla_{3}^{2} \psi}{g_{3}}\right)\right]=} \\
& -i \frac{i}{k_{2}} \frac{\omega}{\bar{\omega}} \sqrt{g_{3}} \widehat{L}_{T}\left(\nabla_{1}-(\ln \bar{\omega})^{\prime}\right) \varphi,
\end{aligned}
$$

where $g=g_{1} g_{2} g_{3}$,

$$
\begin{aligned}
& \widehat{L}_{T}=\frac{1}{\sqrt{g_{3}}} \nabla_{3} \frac{p}{\sqrt{g_{3}}} \nabla_{3}+p \frac{\bar{\omega}^{2}}{A^{2}}, \\
& \widehat{L}_{P}=\frac{1}{\sqrt{g_{3}}} \nabla_{3} \frac{p^{-1}}{\sqrt{g_{3}}} \nabla_{3}+p^{-1} \frac{\bar{\omega}^{2}}{A^{2}},
\end{aligned}
$$

are the toroidal and poloidal longitudinal operators, $p=\sqrt{g_{2} / g_{1}}$,

$$
\begin{aligned}
\widehat{L}_{F 0}= & \nabla_{1} \frac{\bar{\omega}}{\omega} \widehat{L}_{T} \frac{g_{1}}{\sqrt{g}}-\widehat{L}_{P} \frac{g_{2}}{\sqrt{g}} \nabla_{1}, \\
\widehat{L}_{F 1}= & \nabla_{1} \widehat{L}_{T} \frac{\sqrt{g}}{g_{3}}\left(\frac{\Omega}{\omega}\left(\frac{\nabla_{1}^{2} \Psi}{g_{1}}+\frac{\nabla_{3}^{2} \Psi}{g_{3}}\right)+\frac{\Omega^{\prime}}{\bar{\omega}} \frac{\nabla_{1} \Psi}{g_{1}}\right) \\
& +i \frac{k_{2}^{2}}{\omega} \frac{S^{2}}{C_{s}^{2}} \frac{\Omega^{\prime}}{\sqrt{g_{3}}}\left(\frac{\nabla_{1}^{2} \Psi}{g_{1}}-\frac{k_{2}^{2}}{g_{2}} \Psi-\frac{k_{2} \Omega}{\bar{\omega}} \frac{\nabla_{3}^{2} \Psi}{g_{3}}\right),
\end{aligned}
$$

$A=B_{0} / \sqrt{4 \pi \rho_{0}}$ is the Alfvén velocity, $S=\sqrt{\gamma P_{0} / \rho_{0}}$ is the sound velocity in plasmas, $C_{S}=A S / \sqrt{A^{2}+S^{2}}$ is the velocity of slow magnetosonic waves.

We assume that the plasma motion exerts a special influence on the resonant Alfvén wave structure in the regions with a high velocity gradient $\nabla_{1} \Omega\left(x^{1}\right) \equiv \Omega^{\prime}$. In this case, in terms proportional to $\Omega$, the derivatives of the medium parameters are ignored, except for $\Omega^{\prime}\left(x^{1}\right)$. In a similar manner, no account is taken of the terms with the derivatives of medium parameters proportional to the sound velocity $S$ (where $S \ll A$ ). Besides, we employ the following equation relating the potentials $\varphi, \psi$ and $\xi$

$\frac{g_{3}}{\sqrt{g}} \nabla_{1} \xi=\nabla_{3}\left(\frac{\omega}{\bar{\omega}} \varphi-i \frac{\Omega}{\bar{\omega}} \frac{g_{2}}{\sqrt{g}} \nabla_{1} \Psi\right)$,

obtained from the third Eq. (7). If plasma is cold $\left(P_{0}=0\right)$ and stationary $(\Omega=0)$, Eqs. (8), (9) transform into corresponding equations describing the structure of resonant Alfvén and FMS oscillations in cold plasma (Leonovich, 2001).

It is easy to verify that the right sides of Eqs. (8) and (9) become zero in homogenous motionless plasma. The left side 
of Eq. (8) yields a dispersion equation for Alfvén waves: $\omega^{2}-k_{\|}^{2} A^{2}=0$; while the left side of Eq. (9) gives such an equation for fast magnetic sound: $\omega^{2}-k^{2}\left(A^{2}+S^{2}\right)=0$. Here $k^{2}=k_{\|}^{2}+k_{\perp}^{2}$ is the square of the total wave vector, $k_{\|}$and $k_{\perp}$ are its parallel and perpendicular components about the $\mathbf{B}_{\mathbf{0}}$. In inhomogeneous plasma, these oscillations are interrelated near resonant surfaces, where the magnetosonic wave frequency is equal to the local frequency of Alfvén oscillations. Further calculations will prove that the oscillation field related to the scalar potential $\varphi$ dominates near the resonant surface. Its polarization corresponds to Alfvén waves. If regularizing factors (such as dissipation or finite viscosity) are ignored, the $\varphi$ field has a singularity on the resonant surface. Hence the oscillations associated with the potential $\varphi$ can be interpreted as Alfvén oscillations. The amplitude of these oscillations tends to decrease with distance from the resonant surface. The scale of this decrease is determined by a regularizing factor. Oscillations associated with the potential $\psi$ prevail far from this surface. The dispersion and polarization of these oscillations correspond to the fast magnetic sound.

In Eq. (8), the right side describing FMS oscillations acts as a source of resonant Alfvén waves. The right side of Eq. (9) can be interpreted as a feedback from the Alfvén wave on the field of FMS oscillations. As was demonstrated in Leonovich (2001), this influence is insignificant and can be ignored in a zeroth-order approximation. Thus, the field of monochromatic FMS wave can be described by Eq. (9) with a zero right side. Studying this equation in moving plasma is a complicated problem that is beyond the scope of our task. From here on we will suppose that the FMS wave field is specified by a solution of the homogeneous Eq. (9).

Let us note an important peculiarity of this equation. It is well known that, in immobile ideal plasma, the field line resonance may only occur for oscillations with $k_{2} \neq 0$. This agrees with the presence of the factor $k_{2}$, preceding the operator $\widehat{L}_{F 0}$ in the right side of Eq. (8). At first sight, it seems that moving plasma provides an opportunity for resonant oscillations with $k_{2}=0$, since the $\widehat{L}_{F 1}$ operator does not become zero when $k_{2} \rightarrow 0$. However, if $k_{2} \rightarrow 0$ in Eq. (9), and the expression obtained there is taken into account, Eq. (8) becomes zero, identically. Thus, excitation of Alfvén oscillations on the resonant surface is only possible for oscillations with $k_{2} \neq 0$ in both the immovable and moving plasma cases.

We will focus on the Alfvén oscillations in the vicinity of resonant surfaces. Further calculations will show that the potential $\varphi$ has a singularity on these surfaces. The field of resonant Alfvén oscillations can be expressed through it. Let us use the potential $\varphi$ to write down expressions for the components of the velocity and electromagnetic field associated with the Alfvén oscillations:

$$
\begin{aligned}
& E_{1}=-\nabla_{1} \varphi, \quad E_{2}=-i k_{2} \varphi, \quad E_{3}=\frac{k_{2} \Omega}{\bar{\omega}} \nabla_{3} \varphi, \\
& B_{1}=\frac{k_{2} c}{\bar{\omega}} \frac{g_{1}}{\sqrt{g}} \nabla_{3} \varphi,
\end{aligned}
$$

$$
\begin{aligned}
& B_{2}=i \frac{c}{\bar{\omega}} \frac{g_{2}}{\sqrt{g}} \nabla_{3}\left(\nabla_{1}-k_{2} \frac{\Omega^{\prime}}{\bar{\omega}}\right) \varphi, \quad B_{3}=0, \\
& v_{1}=-i \frac{k_{2}}{p} \frac{c}{B_{0}} \varphi, \quad v_{2}=\frac{c p}{B_{0}} \nabla_{1} \varphi, \\
& v_{3} \approx i \frac{c p}{B_{0}} \frac{\Omega}{\bar{\omega}}\left(\nabla_{3} \ln g_{2}\right) \nabla_{1} \varphi .
\end{aligned}
$$

Let us consider Eq. (8) describing the field of resonant Alfvén wave.

\section{The structure of resonant Alfvén waves along geo- magnetic field lines}

The assumption that the structure of the solution across magnetic shells is small-scale permits the method of different scales (see Leonovich and Mazur, 1989) to be used:

$\varphi=V\left(x^{1}\right)\left(T\left(x^{1}, x^{3}\right)+\tau\left(x^{1}, x^{3}\right)\right) \exp \left[i k_{2} x^{2}-i \omega t\right]$,

where the function $V\left(x^{1}\right)$ describes the small-scale solution structure across magnetic shells, $T\left(x^{1}, x^{3}\right)$ presents the solution structure along magnetic field lines (the scale of this function variation in $x^{1}$ is identical to that of the medium's inhomogeneity, while being much larger than in $V\left(x^{1}\right)$ ), $\tau\left(x^{1}, x^{3}\right)$ is the higher order correction of the perturbation theory. As the zero approximation, we derive an equation retaining only the main terms proportional to $\nabla_{1}^{2} V\left(x^{1}\right)$ in Eq. (8):

$\widehat{L}_{T}(\bar{\omega}) T \equiv \frac{\partial}{\partial \ell} p \frac{\partial T}{\partial \ell}+p \frac{\bar{\omega}^{2}}{A^{2}} T=0$,

that determines the structure of the Alfvén oscillation field along geomagnetic field lines. Here $\ell$ is the field line length measured from the equator $\left(d \ell=\sqrt{g_{3}} d x^{3}\right)$. For this approximation, the ionosphere may be considered ideally conductive, yielding boundary conditions $T\left(x^{1}, \ell_{ \pm}\right)=0$ in which the \pm signs refer to the ionospheres of the Northern and Southern Hemisphere, respectively. The eigenfunctions $T_{N}\left(x^{1}, \ell\right)$ and corresponding eigenvalues $\omega=\Omega_{N}$, where $N=1,2,3 \ldots$ is a wave number of the longitudinal harmonic of the oscillations, provide a solution to Eq. (12) with such boundary conditions. These solutions are Alfvén waves standing along geomagnetic field lines and with $N-1$ nodes on the field line. For sufficiently high wave numbers $(N \gg 1)$ the solution may be presented in the WKB approximation. Let us choose normalized conditions for the eigenfunctions as follows:

$$
\int_{\ell_{-}}^{\ell_{+}} \frac{T_{N}^{2} p}{A^{2}} d \ell=1 .
$$

Then, the WKB-approximation solution may be shown as

$$
T_{N}=\sqrt{\frac{2 A}{p t_{A}}} \sin \left(\Omega_{N} \int_{\ell_{-}}^{\ell} \frac{d \ell^{\prime}}{A\left(x^{1}, \ell^{\prime}\right)}\right), \quad \Omega_{N}=\frac{\pi N}{t_{A}},
$$




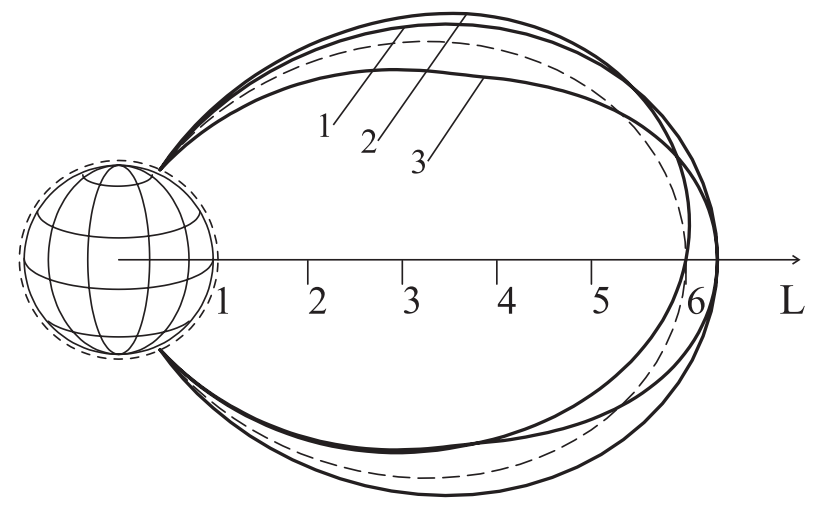

Fig. 2. The structure of the first three harmonics of standing Alfvén waves (bold lines) on the magnetic shell $L=6$ (dashed line).

where

$t_{A}\left(x^{1}\right)=\int_{\ell_{-}}^{\ell_{+}} \frac{d \ell}{A\left(x^{1}, \ell\right)}$

is the travel time at the Alfvén velocity along the field line between the magnetoconjugated ionospheres. Figure 2 demonstrates the structure of the first three harmonics of standing Alfvén waves on the magnetic shell $L=6$ (where $L=a / R_{E}$ is the McIlwain parameter, $a$ is the equatorial radius of the field line (see Fig. 1), $R_{E}$ is the Earth's radius) for a model magnetosphere with dipole magnetic field. Plasma distribution is specified according to the self-consistent model of the magnetosphere with rotating plasma (Leonovich et al., 2004, see Fig. 3).

\section{The transverse structure of resonant Alfvén waves}

Now we will consider the structure of Alfvén oscillations across magnetic shells near the resonant surface. Substitute the solution in the form Eq. (11) into Eq. (8) and consider the solution for the function $T=T_{N}\left(x^{1}, \ell\right)$ obtained from Eq. (12). In the first order of the perturbation theory we derive the following equation:

$$
\begin{aligned}
& \left(\nabla_{1}^{2} V_{N}\right) \frac{\bar{\omega}^{2}-\Omega_{N}^{2}}{A^{2}} p T_{N}+\left(\nabla_{1}^{2} V_{N}\right) \widehat{L}_{T}\left(\Omega_{N}\right) \tau_{N} \\
& \quad+\left(\nabla_{1} V_{N}\right) \nabla_{1} \frac{\bar{\omega}^{2}-\Omega_{N}^{2}}{A^{2}} p T_{N}- \\
& \quad\left(\nabla_{1} V_{N}\right)\left(\nabla_{1} \ln \bar{\omega}\right) \frac{\bar{\omega}^{2}-\Omega_{N}^{2}}{A^{2}} p T_{N}-k_{2}^{2} V_{N} \widehat{L}_{P}(\bar{\omega}) T_{N} \\
& =i k_{2} \hat{L}_{F 0} \psi+i \hat{L}_{F 1} \psi .
\end{aligned}
$$

In the same approximation, the boundary condition on the ionosphere regarding its finite Pedersen conductivity is of the form (Leonovich and Mazur, 1996):

$$
\left.\tau_{N}\right|_{\ell_{ \pm}}=\left.\mp \frac{v_{ \pm}}{\Omega_{N}} \frac{\partial T_{N}}{\partial \ell}\right|_{\ell_{ \pm}},
$$

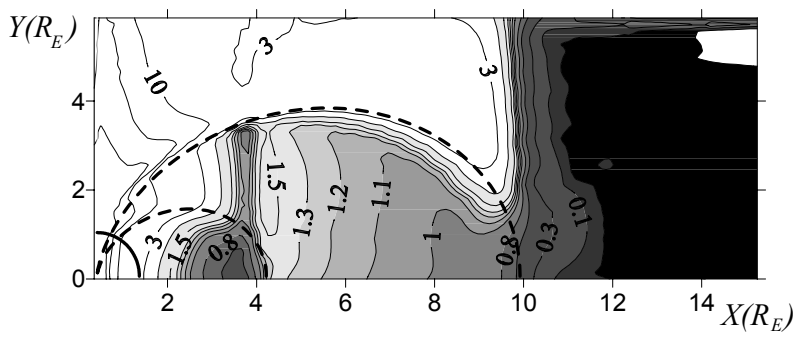

Fig. 3. The Alfvén velocity $\left(10^{3} \mathrm{~km} / \mathrm{s}\right)$ isolines in the magnetic meridian plane in the magnetospheric model under study. The dashed lines indicate the plasmapause $(L=4)$ and magnetopause $(L=10)$.

where

$v_{ \pm}=\frac{c^{2} \cos \chi_{ \pm}}{4 \pi \Sigma_{p}^{ \pm}}$

The signs \pm refer to the Northern and Southern Hemispheres, $\chi$ is the angle between the vertical and the field line at its intersection point with the ionosphere (see Fig. 1), $\Sigma_{p}$ is the integrated Pedersen conductivity in the ionosphere. Multiplying this equation by $T_{N}$ on the left and integrating it along the field line between the magnetoconjugated ionospheres yields

$$
\begin{gathered}
\nabla_{1}\left[\left(\bar{\omega}+i \gamma_{N}\right)^{2}-\Omega_{N}^{2}\right] \nabla_{1} V_{N}-\left(\bar{\omega}^{2}-\Omega_{N}^{2}\right)\left(\nabla_{1} \ln \bar{\omega}\right) \nabla_{1} V_{N} \\
-k_{2}^{2}\left[\alpha_{N}+\bar{\alpha}_{N}\left(\left(\bar{\omega}+i \gamma_{N}\right)^{2}-\Omega_{N}^{2}\right)\right]=i \mu_{N},
\end{gathered}
$$

where

$$
\begin{aligned}
& \alpha_{N}=\int_{\ell_{-}}^{\ell_{+}} T_{N} \widehat{L}_{P}\left(\Omega_{N}\right) T_{N} d \ell=-\int_{\ell_{-}}^{\ell_{+}} T_{N}^{2} \frac{\partial^{2} p^{-1}}{\partial \ell^{2}} d \ell, \\
& \bar{\alpha}_{N}=\int_{\ell_{-}}^{\ell_{+}} \frac{p^{-1} T_{N}^{2}}{A^{2}} d \ell, \\
& \mu_{N}=\int_{\ell_{-}}^{\ell_{+}} T_{N}\left(k_{2} \hat{L}_{F 0} \psi+\hat{L}_{F 1} \psi\right) d \ell .
\end{aligned}
$$

An imaginary addition to the frequency $i \gamma_{N}$ (oscillation decrement) that emerges from the boundary conditions (Eq. 15), when we integrate the term with $\tau_{N}$ by parts, is:

$$
\gamma_{N}=\frac{1}{\bar{\omega}^{2}}\left[p_{+} v_{+}\left(\frac{\partial T_{N}}{\partial \ell}\right)_{\ell_{+}}^{2}+p_{-} v_{-}\left(\frac{\partial T_{N}}{\partial \ell}\right)_{\ell_{-}}^{2}\right] \text {. }
$$

For the function $\Omega_{N}\left(x^{1}\right)$ near the resonant surface $x^{1}=x_{T N}^{1}$ $\left(\Omega_{N}\left(x_{T N}^{1}\right)=\bar{\omega}\right)$, we employ the linear decomposition

$$
\Omega_{N}^{2} \approx \bar{\omega}^{2}\left(1-\frac{x^{1}-x_{T N}^{1}}{2 \bar{a}}\right) \text {, }
$$




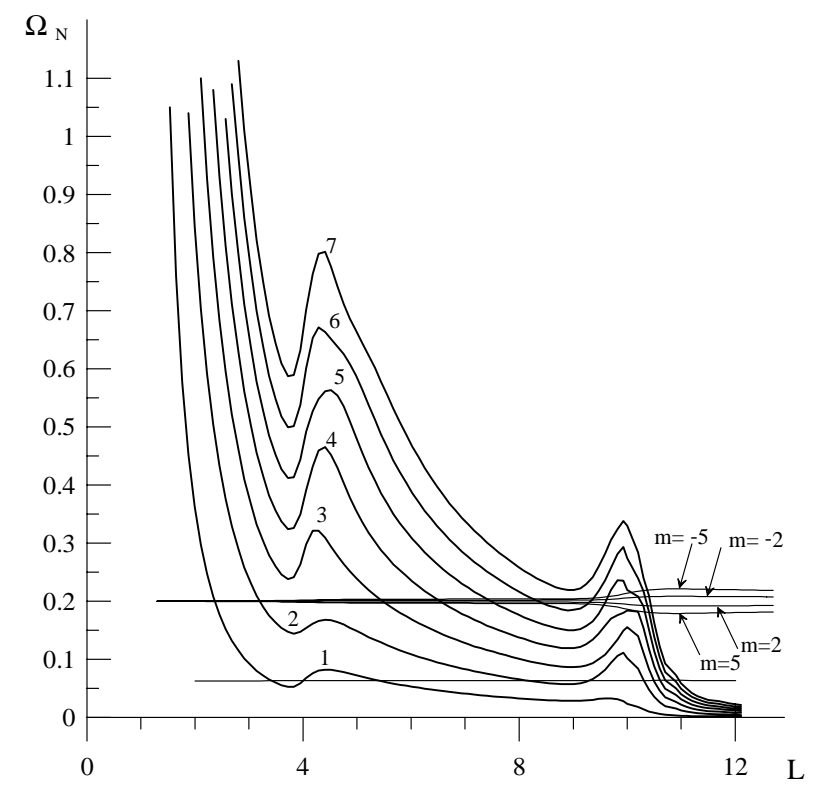

Fig. 4. The distribution of eigenfrequencies of the first harmonics of standing Alfvén waves $(N=1,2, \ldots, 7)$ across magnetic shells and the value $\bar{\omega}=\omega-m \Omega\left(x^{1}\right)$ for $f=\omega / 2 \pi=10^{-2} \mathrm{~Hz}$ and $m= \pm 2,5$. The intersection points of these functions specify the location of resonant surfaces.

where $\bar{a}=\left[\left(\ln \Omega_{N}\left(x^{1}\right)\right)^{\prime}\right]^{-1}$ is the typical scale of plasma inhomogeneity. Introducing a dimensionless coordinate $\xi=\left(x^{1}-x_{T N}^{1}\right) / \bar{a}$, we bring Eq. (16) into the form

$$
\begin{gathered}
\frac{\partial}{\partial \xi}(\xi+i \varepsilon) \frac{\partial V_{N}}{\partial \xi}+d_{N}(\xi+i \varepsilon) \frac{\partial V_{N}}{\partial \xi} \\
-\kappa_{N}^{2}\left[1+\beta_{N}(\xi+i \varepsilon)\right]=i f_{N},
\end{gathered}
$$

where $\varepsilon=\gamma_{N} / \bar{\omega}, f_{N}=\bar{a}^{2} \mu_{N} / \bar{\omega}^{2}$,

$d_{N}=\frac{\Omega^{\prime}}{\Omega_{N}} k_{2} \bar{a}, \quad \kappa_{N}^{2}=\frac{\alpha_{N}}{\Omega_{N}^{2}} k_{2}^{2} \bar{a}^{2}, \quad \beta_{N}=\frac{\bar{\alpha}_{N}}{\alpha_{N}} \Omega_{N}^{2}$.

We will solve this equation with the Fourier-transformation method representing $V_{N}(\xi)$ as

$V_{N}(\xi)=\frac{1}{\sqrt{2 \pi}} \int_{-\infty}^{\infty} \tilde{V}_{N}(k) \exp (i k \xi) d k$.

Substituting this into Eq. (17), we derive an easy-to-solve, first-order differential equation for $\tilde{V}_{N}$. Returning to the solution $V_{N}(\xi)$, we deduce the following:

$V_{N}(\xi)=-\frac{f_{N}}{\kappa_{N} \sqrt{\beta_{N}}} \int_{0}^{\infty} \frac{\exp [i k(\xi+i \varepsilon)+i \Psi(k)]}{\sqrt{k^{2}-i d_{N} k+\kappa_{N}^{2} \beta_{N}}} d k$,

where

$\Psi(k)=\frac{\kappa_{N}^{2}+d_{N} / 2}{\sqrt{\kappa_{N}^{2} \beta_{N}+d_{N}^{2} / 4}} \arctan \frac{k \sqrt{\kappa_{N}^{2} \beta_{N}+d_{N}^{2} / 4}}{\kappa_{N}^{2} \beta_{N}-i k d_{N} / 2}$.
Let us discuss the behaviour of the solution obtained here near the resonant shell $\xi=0$ and in the asymptotic region $|\xi| \rightarrow \infty$. Near the resonant surface, the bulk of the integral (18) is composed with $k \rightarrow \infty$. Making $k \rightarrow \infty$ in the integrand denominator yields

$$
\begin{aligned}
\frac{\partial V_{N}}{\partial \xi} & \approx \frac{f_{N}}{\kappa_{N} \sqrt{\beta_{N}}} \frac{\exp [i \Psi(\infty)]}{\xi+i \varepsilon}, \\
V_{N} & \approx \frac{f_{N}}{\kappa_{N} \sqrt{\beta_{N}}} \exp [i \Psi(\infty)] \ln (\xi+i \varepsilon) .
\end{aligned}
$$

It is evident that the field of the resonant Alfvén wave has a well-known singularity when $\varepsilon \rightarrow 0$.

Conversely, at the asymptotics $|\xi| \rightarrow \infty$, the bulk of the integral (18) is due to small values of $k \rightarrow 0$. Assuming $k=0$ in the integrand denominator and in $\Psi(k)$, we have

$$
V_{N} \approx-i \frac{f_{N}}{\kappa_{N} \sqrt{\beta_{N}}} \frac{1}{\xi+i \varepsilon} .
$$

The resonant oscillation field linearly decreases with distance from the resonant surface on a scale $\sim\left|\left(\gamma_{N} / \bar{\omega}\right) \bar{a}\right| \ll \bar{a}$.

\section{Discussion}

Let us numerically investigate the resonant Alfvén oscillations excited by the monochromatic FMS wave in the magnetosphere with dipole magnetic field. Figure 3 presents the Alfvén velocity distribution in the meridional plane in this model (see Leonovich et al., 2004). This model simulates the dayside part of the moderately disturbed magnetosphere fairly well. For numerical calculations we employed the nonorthogonal coordinate system $(a, \phi, \theta)$ in which the equatorial radius of the magnetic shell $a$, the azimuthal angle $\phi$, and the latitude $\theta$, measured from the equator, are unambiguously related to the transverse coordinate $x^{1}$, the azimuthal coordinate $x^{2}$, and the longitudinal coordinate $x^{3}$ respectively (see Fig. 1). In this coordinate system

$$
\begin{aligned}
& d \ell \equiv \sqrt{g_{3}} d x^{3}=a \cos \theta \sqrt{1+3 \sin ^{2} \theta} d \theta, \\
& g_{1}=\cos ^{6} \theta /\left(1+3 \sin ^{2} \theta\right), \quad g_{2}=a^{2} \cos ^{6} \theta .
\end{aligned}
$$

Figure 4 shows the distribution of several first eigenfrequencies of standing Alfvén waves across magnetic shells $\Omega_{N}\left(x^{1}\right)$. Also presented is the distribution of functions $\bar{\omega}\left(x^{1}\right)$ for several values of the azimuthal wave number $m$ and for the fixed frequency of magnetosonic oscillations $f=\omega / 2 \pi=10^{-2} \mathrm{~Hz}$. Intersection points of these functions are resonant surface positions. Obviously the resonant surfaces are mainly concentrated in the transition zone of the magnetosphere, near the magnetopause.

We denote a physical component of the magnetic field of Alfvén oscillations that has the highest amplitude on the resonant magnetic shell as $B_{y}=B_{2} / \sqrt{g_{2}}=\left|B_{y}\right| \exp \left(i \alpha_{y}\right)$. As evident from test calculations, the best effect of the background 
(a)

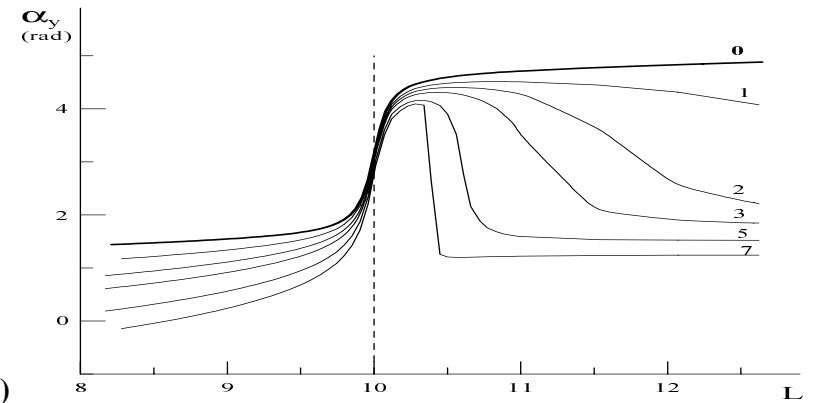

(b)

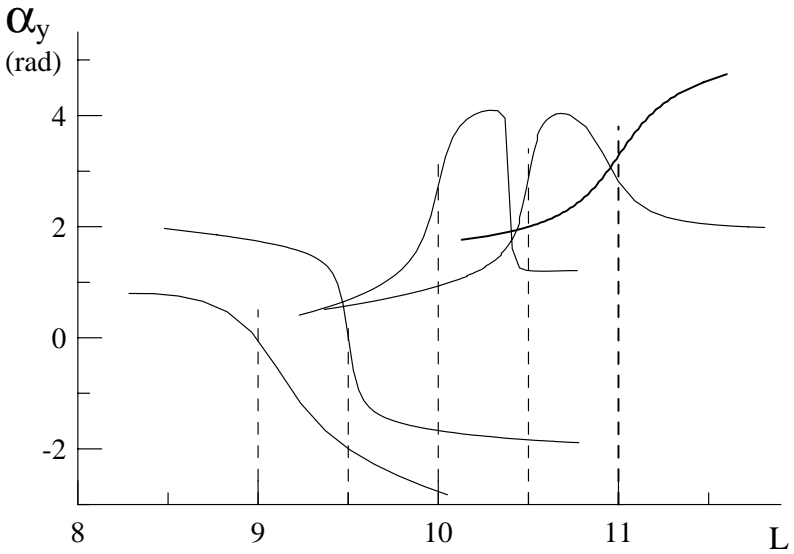

Fig. 5. The distribution of the resonant Alfvén oscillation phase $\alpha_{y}$ across magnetic shells. Panel (a) presents the distribution of $\alpha_{y}\left(x^{1}\right)$ in the model with moving plasma on the magnetic shell $L=10$ for the harmonics $N=1, m=1,2,3,5,7$, as well as in the case of stationary plasma $(\Omega=0)$ (that is similar to the choice of $m=0$ ). Panel (b) displays the distribution of $\alpha_{y}\left(x^{1}\right)$ for the harmonic $N=1, m=7$ on different resonant shells within the transition layer.

plasma motion manifests itself in the transition zone near the magnetopause. Let us consider the resonant oscillation phase $\alpha_{y}$ distribution across the resonant shell $L=10$, where the gradient $\nabla_{1} \Omega\left(x^{1}\right)$ is maximum (Fig. 5a). The plasma motion effect increases significantly with the increasing number of the azimuthal harmonic of the oscillations. This implies that the phase $\alpha_{y}$ varies nonmonotonically across the resonant layer, and decreases after having increased by $\sim \pi$. The higher the number is of the azimuthal harmonic $m$, the smaller the scale of its subsequent decrease. This scale increases with an increasing in the number of the longitudinal harmonic $N$. Figure $5 \mathrm{~b}$ presents the distribution $\alpha_{y}\left(x^{1}\right)$ for the harmonic $N=1, m=7$ on different resonant magnetic shells within the transition layer $9<L<11$. The higher the plasma motion velocity gradient, the more pronounced its effect on the phase of resonant oscillations. Notice that near the plasmapause the same effect may be observed only for the first harmonics of the resonant Alfvén oscillations with a rather thin plasmapause $\left(\sim 0.1 R_{E}\right)$, when the gradient $\nabla_{1} \Omega\left(x^{1}\right)$ is comparable with that on the magnetopause. (a)
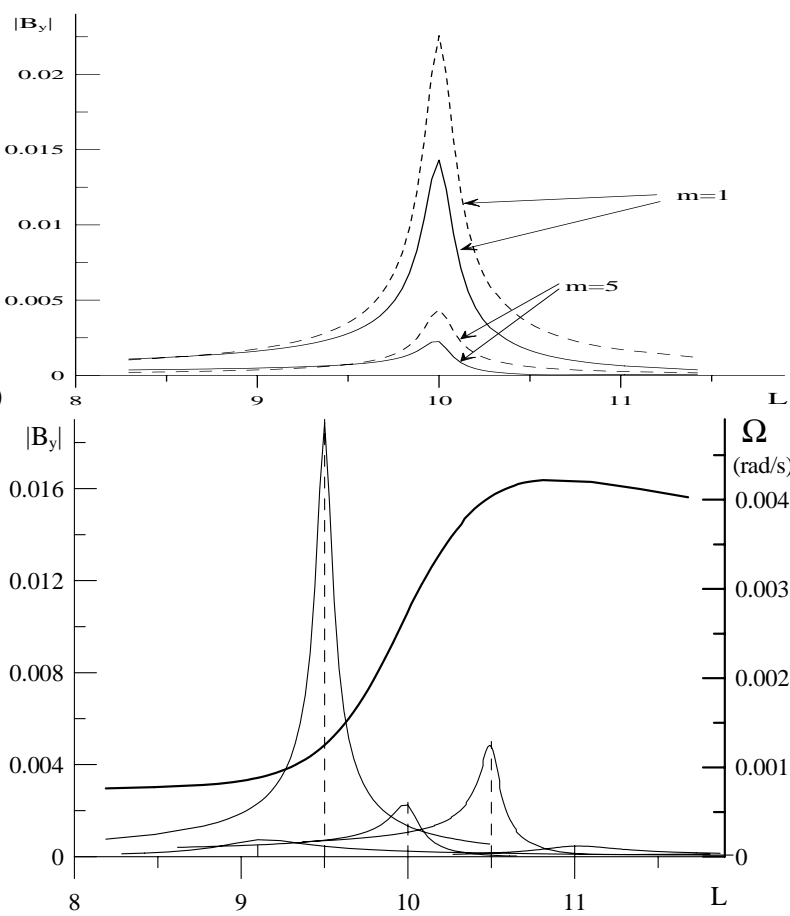

Fig. 6. The distribution of the resonant Alfvén oscillation amplitude $\left|B_{y}\right|$ across magnetic shells. Panel (a) shows the distribution of $\left|B_{y}\left(x^{1}\right)\right|$ on the magnetic shell $L=10$ for the harmonics $N=1, m=1,5$, both in the moving plasma model (solid lines) and in the stationary plasma case (dashed lines). Panel (b) presents the distribution of $\left|B_{y}\left(x^{1}\right)\right|$ inside the transition layer for the harmonic $N=1, m=7$, on different resonant shells (left semiaxis), as well as of the plasma angular velocity $\Omega\left(x^{1}\right)$ (right semiaxis).

Nonmonotonic behaviour of the phase of resonant Alfvén oscillations can be an indicator of a region with a high gradient of the background plasma velocity.

Figure 6 presents similar distributions of the resonant oscillation amplitude $\left|B_{y}\right|$ within the transition layer. In these calculations, we used the solution of Eq. (17) with the unity right side $f_{N}=1$. Hereafter the calculations are performed with a sufficiently small decrement of oscillations $\gamma_{N}=10^{-3} \mathrm{~s}^{-1}$, in order to reveal their resonant structure. Figure 6a shows the amplitude distribution of the basic longitudinal harmonic of resonant oscillations $N=1$ excited by FMS waves with different azimuthal wave numbers $m=1,5$ on the magnetic shell $L=10$. The plasma motion effect also increases here with increasing $m$, though it is less pronounced than for the phase of the oscillations considered. This effect is an amplitude profile asymmetry. In the same figure, for comparison, the amplitude distributions are plotted without the plasma motion (i.e. with $\Omega=0$ ) of the same resonant oscillations, which are symmetric about the resonant surface. Figure $5 \mathrm{~b}$ shows amplitude profiles of the resonant harmonic $N=1, m=5$ on different magnetic shells inside the transition 
(a)

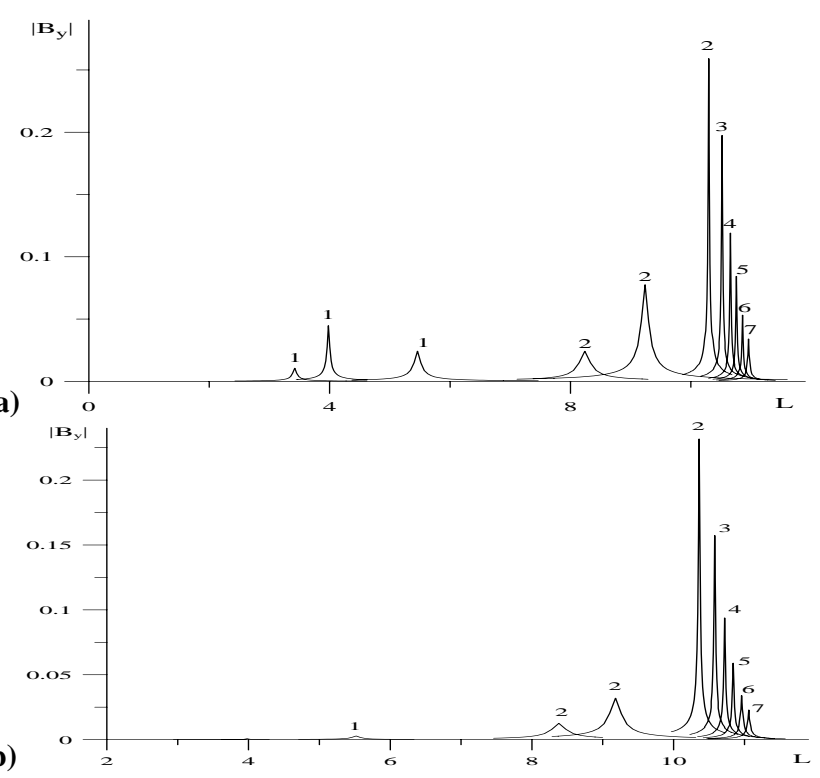

Fig. 7. The distribution of the amplitude (in the ionospheric projection) of the total field of resonant oscillations excited by the monochromatic FMS wave (by the model driver (19)) in the dipole magnetosphere. Panel (a) shows the field of oscillations excited by the FMS harmonic $n=1, m=1$ with a frequency of $0.01 \mathrm{~Hz}$; Panel (b) presents the field of oscillations excited by the harmonic $n=5, m=3$ with the same frequency.

layer. The angular velocity of the plasma rotation profile $\Omega\left(x^{1}\right)$ is also presented. It is obvious that the largest asymmetry of the amplitude distribution can be observed in the region with a maximum gradient value $\nabla_{1} \Omega\left(x^{1}\right)$.

Let us consider now the total field of magnetospheric resonant oscillations, which can be excited by the monochromatic FMS wave that penetrates into the magnetosphere from the solar wind or is induced by the Kelvin-Helmholtz instability on the magnetopause. An investigation of magnetosonic oscillations in the magnetospheric model under study is a rather complicated problem that requires special examination. In this paper, we will replace the real solution of Eq. (9) with the model driver using certain peculiarities of the field of low-frequency FMS waves in the dipole magnetospheric model (see Leonovich and Mazur, 2000). Firstly, the inner magnetosphere is an opacity region for such oscillations. As the WKB approximation, the typical scale of an FMS oscillation amplitude decrease is determined by a factor $\exp \left[-\int\left|k_{1}\right| d x^{1}\right]$, where the wave vector component $k_{1}$ is defined by the local dispersion equation $k_{1}^{2}\left(x^{1}\right)=\omega^{2} /\left(A^{2}+S^{2}\right)-k_{2}^{2} / g_{2}-k_{3}^{2} / g_{3}$. For extremely lowfrequency waves $k_{1}^{2}\left(x^{1}\right) \approx-k_{2}^{2} / g_{2}-k_{3}^{2} / g_{3}$; i.e. the inner magnetosphere is the opacity region for them. Therefore we will

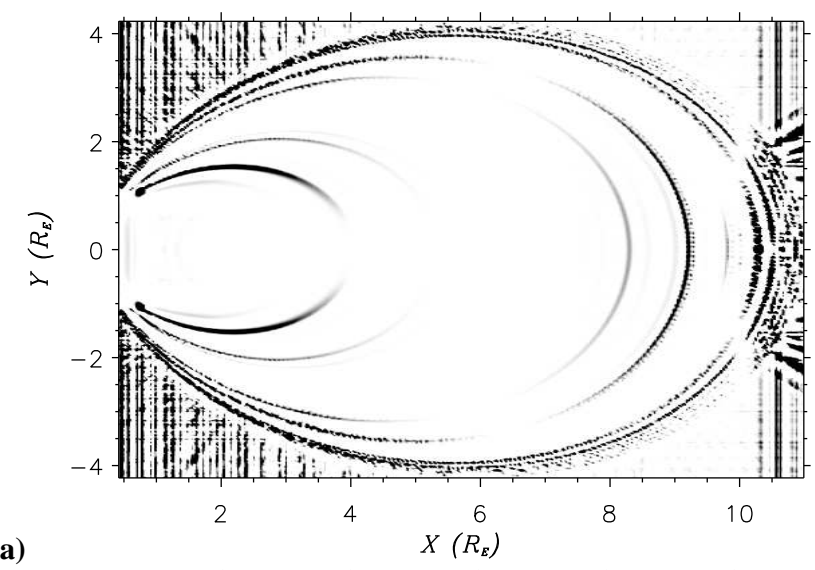

(a)

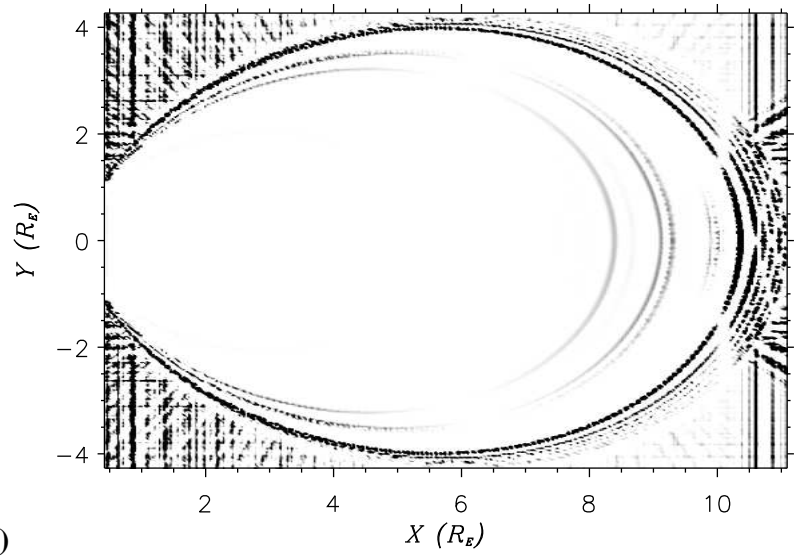

Fig. 8. The distribution of the amplitude $\left|B_{y}\left(x^{1}\right)\right|$ of the same oscillations as in Fig. 7, in the meridional plane. The darker colour marks the regions with high oscillation amplitude.

employ the following model for the function:

$f_{N}=m \exp \left[-\sqrt{\frac{m^{2}}{a^{2}}+\frac{n^{2}}{L^{2}}}\left(a_{m p}-a\right)\right]$,

where $m$ and $n$ are azimuthal and longitudinal wave numbers for the harmonic of FMS oscillations, $a$ and $L$ are the typical equatorial radius and length of the field line of the resonant magnetic shell in question, $a_{m p}$ is the equatorial radius of the magnetopause. The factor $m$ preceding the exponent corresponds to the factor $k_{2}$ preceding the operator $\hat{L}_{F 0}$ in Eq. (8).

Figure 7 a presents the distribution of the amplitude of resonant Alfvén oscillations excited by the harmonic of FMS oscillations $n=1, m=1$ with a frequency of $0.01 \mathrm{~Hz}$, across magnetic shells. One can see that the main three harmonics of standing Alfvén waves $N=1$, the second three harmonics $N=2$, and higher harmonics are excited, respectively, inside the inner magnetosphere, in the outer magnetosphere and in the transition layer region. Figure $7 b$ shows the field of resonant Alfvén oscillations excited by the FMS harmonic $n=5, m=3$ with the same frequency of $0.01 \mathrm{~Hz}$. Whereas 
the oscillation amplitude in the transition layer is practically the same as in the previous case, the resonant oscillations abruptly lose their amplitude away from the magnetopause inside the magnetosphere. Figure 8a, b shows a distribution of the same resonant oscillations in the magnetic meridian plane. It is evident from these figures that the resonant oscillation amplitude peaks near the equatorial plane (in longitudinal structure antinodes) and ionosphere. The latter can be explained by the abrupt increase in the Alfvén velocity value as the ionosphere is approached (see Eq. 14).

\section{Conclusion}

Let us list the main findings of our investigation.

1. A system for Eqs. (8-9) was derived describing the relationship between Alfvén and magnetosonic oscillations in a dipole magnetosphere with rotating plasma.

2. Analytical solutions were found to Eq. (8) which describe the structure of standing Alfvén waves excited by the field of the monochromatic FMS wave on resonant magnetic shells.

3. A numerical investigation of the field of resonant Alfvén waves excited by a driver simulating a low-frequency FMS wave was performed. The background plasma motion effect was demonstrated to be maximum in a region with the maximum gradient of its velocity. These regions are in the transition layer near the magnetopause and at the plasmapause (when it is fairly thin: $\sim 0.1 R_{E}$ ). This effect implies that the phase of resonant oscillations varies nonmonotonically in the vicinity of the resonant magnetic shell, and their amplitude profile becomes asymmetrical about the resonant surface. It can serve as an indicator of regions with a high gradient of the background plasma velocity.

4. A model with a smooth transition layer and straight magnetic field lines has only one resonant surface for the monochromatic FMS wave. In a magnetospheric model with curved field lines there are numerous resonant surfaces for resonant Alfvén oscillations. The highest density of resonant surfaces can be observed in the transition layer near the magnetopause. Due to the complicated profile of the Alfvén velocity there may be several resonant surfaces for the same harmonic of standing Alfvén waves inside the magnetosphere. Resonant oscillations excited by the basic (i.e. the largestscale) FMS wave field harmonics have the highest amplitude inside the magnetosphere.

Acknowledgements. This work was partially supported by grants 06-05-64495, 07-05-00185, from the Russian Foundation for Basic Research and by the Program of presidium of the Russian Academy of Sciences \#16 and OFN RAS \#16.
Topical Editor I. A. Daglis thanks one anonymous referee for her/his help in evaluating this paper.

\section{References}

Allan, W., White, S. P., and Poulter, E. M.: Impulse-excited hydromagnetic cavity and field line resonance in the magnetosphere, Planet. Space Sci., 34, 371-380, 1986.

Allan, W., Poulter, E. M., and White, S. P.: Structure of magnetospheric MHD resonances for moderate "azimuthal" asymmetry, Planet. Space Sci., 35, 1193-1198, 1987.

Chen, L. and Hasegawa, A.: A theory of long period magnetic pulsation. 1. Steady state excitation of field line resonances, J. Geophys. Res., 79, 1024-1032, 1974.

Dungey, J. W.: Electrodynamics of the outer atmospheres, Ionos. Sci. Rep., 69, Ionos. Res. Lab., Cambridge, Pa., 1954.

Chen, L. and Cowley, S. C.: On field line resonances of hydromagnetic Alfvén waves in a dipole magnetic field, Geophys. Res. Lett., 16, 895-897, 1989.

Goertz, C. K.: Kinetic Alfvén waves on auroral field lines, Planet. Space Sci., 32, 1387-1392, 1984.

Hasegawa, A.: Particle acceleration by MHD surface wave and formation of aurorae, J. Geophys. Res., 81, 5083-5090, 1976.

Kivelson, M. G. and Southwood, D. J.: Coupling of global magnetospheric MHD eigenmodes to field line resonances, J. Geophys. Res., 91, 4345-4351, 1986.

Korn, G. A. and Korn, T. M.: Mathematical handbook for scientists and engineers, McGraw-Hill Book Company, 1968.

Lee, D.-H. and Lysak, R. L.: Monochromatic ULF wave coupling in the dipole model: The impulsive excitation, J. Geophys. Res., 94, 17 097-17 109, 1989.

Lee, D. H., Lysak, R. L., and Song, Y.: Field line resonances in a nonaxisymmetric magnetic field, J. Geophys. Res., 105, $10703-$ 10711, 2000.

Leonovich, A. S. and Mazur, V. A.: Resonance excitation of standing Alfvén waves in an axisymmetric magnetosphere (monochromatic oscillations), Planet. Space Sci., 37, 10951108, 1989.

Leonovich, A. S. and Mazur, V. A.: Penetration to the Earth's surface of standing Alfvén waves excited by external currents in the ionosphere, Ann. Geophys., 14, 545-556, 1996, http://www.ann-geophys.net/14/545/1996/.

Leonovich, A. S. and Mazur, V. A.: Structure of magnetosonic eigenoscillations of an axisymmetric magnetosphere, J. Geophys. Res., 105, 27 707-27 716, 2000.

Leonovich, A. S.: A theory of field line resonance in a dipole-like axisymmetric magnetosphere, J. Geophys. Res., 106, $25803-$ $25812,2001$.

Leonovich, A. S., Mazur, V. A., and Cao, J. B.: Self-consistent model of a dipole-like mrgnetosphere with an azimuthal solar wind flow, J. Plasma Phys., 70, 99-111, 2004.

Leonovich, A. S. and Mishin, V. V.: Stability of magnetohydrodynamic shear flows with and without bounding walls, J. Plasma Phys., 71, 645-664, 2005.

Lifshitz, A. E. and Fedorov, E. N.: Hydromagnetic oscillations of the ionospheric-magnetospheric resonator, Doklady AN SSSR, 287, 90-95, 1986 (in Russian).

Lysak, R. L. and Song, Y.: Kinetic theory of the Alfvén wave acceleration of auroral electrons, J. Geophys. Res., 108, 8005, 
doi:10.1029/2002JA009406, 2003.

McKenzie, J. F.: Hydromagnetic wave interaction with the magnetopause and the bow shock, Planet. Space Sci., 18, 1-23, 1970.

Radoski, H. R.: A theory of latitude dependent geomagnetic micropulsations: the asymptotic fields, J. Geophys. Res., 79, 595604, 1974.

Samson, J. C., Cogger, L. L., and Pao, Q.: Observations of field line resonances, auroral arcs, and auroral vortex structures, J. Geophys. Res., 101, 17 373-17383, 1996.

Southwood, D. J.: Some features of field line resonances in the magnetosphere, Plahet. Space Sci., 22, 483-492, 1974.
Southwood, D. J. and Kivelson, M. G.: The effect of parallel inhomogeneity of magnetospheric hydromagnetic wave coupling, J. Geophys. Res., 91, 6871-6877, 1986.

Tamao, T.: Transmission and coupling resonance of hydromagnetic disturbances in the non-uniform Earth's magnetosphere, Sci. Rep. Tohoku Univ, ser. 5, 17, 43-72, 1965.

Walker, A. D. M.: Excitation of field line resonances by sources outside the magnetosphere, Ann. Geophys., 23, 3375-3388, 2005, http://www.ann-geophys.net/23/3375/2005/. 